\title{
CBT for major depression may continue to reduce relapse in the long term
}

Fava GA, Rafanelli C, Grandi S, et al. Six-year outcome for cognitive behavioral treatment of residual symptoms in major depression. Am J Psychiatry 1998 Oct;155:1443-5.

\begin{abstract}
Question
In patients who have been successfully treated for major depressive disorder and whose antidepressant doses are being tapered, is cognitive behavioural therapy (CBT) better than standard clinical management $(\mathrm{CM})$ for preventing relapse?
\end{abstract}

\section{Design}

Randomised controlled trial with 6 years of follow up.

\section{Setting}

\{An affective disorders programme in Bologna, Italy. $\}^{*}$

\section{Patients}

\{43 patients were enrolled, and $40(93 \%)$ were analysed (mean age 46 y, 68\% women). .* Patients met the Research Diagnostic Criteria (RDC) for primary major depression and were treated for $\geqslant 3$ months with full doses of antidepressants. \{Exclusion criteria were an unsuccessful response to antidepressants; current medical illness; and history of manic, hypomanic, or cyclothymic features; substance abuse; personality disorder; or antecedent dysthymia. $\}^{*}$

\section{Intervention}

\{After pharmacological treatment $\} *$, patients were allocated to CBT $(n=20)$ or standard CM $(n=20)$. Antidepressants were tapered at the rate of $25 \mathrm{mg}$ of amitriptyline (or its equivalent) every other week $\} *$ and stopped. $\{$ CBT and CM consisted of 10 sessions of 40 minutes once every other week. ${ }^{*}$ Further psychotherapy, drugs, or both, were not permitted during follow up unless a relapse occurred. \{A psychiatrist treated patients in the CBT group and used techniques to help them correct their distorted views and maladaptive beliefs. CM consisted of monitoring drug tapering, reviewing the patient's clinical status, and providing support and advice, if necessary, without using expo- sure strategies, diary work, or cognitive restructuring. Treatment integrity was tested by taping 4 randomly selected sessions for each group; 2 independent assessors correctly identified all 8 sessions. $\}^{*}$

\section{Main outcome measure}

Relapse according to RDC criteria for major depression.

\begin{abstract}
Main results
After 6 years, fewer patients in the CBT group than in the CM group had relapsed, but the results were not statistically significant (log rank test $\mathrm{p}=0.06$ ) (table). Patients in the CBT group had fewer new episodes of depression during follow up than did those in the CM group (mean number of new depressive episodes $0.80 v 1.70, \mathrm{p}<0.05)$.
\end{abstract}

\section{Conclusions}

In patients who were treated successfully with antidepressants for major depression and whose antidepressant doses were being tapered, cognitive behavioural therapy (CBT) may lead to a reduction in the number of patients who relapsed in the long term. CBT led to a reduction in the total number of new depressive episodes during follow up.

*Fava GA, Grandi S, Zielezny M, et al. Am J Psychiatry 1994;151:1295-9.

$C B T v$ CM for major depression treated with antidepressantst

\begin{tabular}{lllll}
\hline Outcome at 6 years & CBT & CM & RRR (95\% CI) & NNT (CI) \\
\hline Relapse & $50 \%$ & $75 \%$ & $33 \%(-9$ to 62$)$ & Not significant
\end{tabular}

$\overline{\dagger \mathrm{CBT}=\text { cognitive behavioural therapy; } \mathrm{CM}=\text { clinical management. Other }}$ abbreviations defined in glossary; RRR, NNT, and CI calculated from data in article.

Source of funding: in part, Istituto Superiore di Sanità.

For correspondence:Dr G A Fava, Department of Psychology, University of Bologna, Viale Berti Pichat 5, 40127 Bologna, Italy. Fax +39051243086.

\section{Commentary}

A high proportion of people treated for major depression will either fail to respond or will relapse within 1 to 2 years. For example, the National Institute of Mental Health's depression study found that after 18 months only $24 \%$ of the original patients had recovered and remained well. ${ }^{1}$ We must treat much depression as a chronic illness, and researchers are trying to understand what helps in its long term management. Fava et al, members of an Italian research group, have concentrated on using CBT to address residual symptoms after treating the patient in the acute episode with drugs. This approach differs from treating the initial episode with CBT or other psycho- logical therapy, or following up an initial psychological intervention with a maintenance treatment. ${ }^{23}$ All of these interventions show promise as ways to improve long term care of people with depression, ${ }^{4}$ although it has not yet been shown that results are attributable to the specific effects of CBT.

The article published in the $A m J$ Psychiatry is the latest of 3 reports about the same cohort of patients at 2, 4, and 6 years after treatment. These patients recovered after antidepressant medication and then residual problems (such as irritability and anxiety) were addressed with CBT. The authors found that this brief intervention reduced the number of residual symptoms, but at 2 years of follow up the relapse rate was not substantially different from routine CM. At 4 years, the CBT group was doing substantially better. The current study reports that at 6 years after treatment, the overall relapse rate difference between the 2 groups was again not statistically significant, although the number of relapses was lower in the CBT group. This pattern of results is difficult to interpret. The series of studies suggests that CBT for residual symptoms may benefit patients; either the benefit fades after 4 years or the sample size is too small to detect a real effect. Both possibilities are equally plausible, and perhaps (commentary continued on page 45) 


\title{
CBT was effective at 2 years in recurrent depression
}

\author{
Fava GA, Rafanelli C, Grandi S, et al. Prevention of recurrent depression with cognitive behavioral therapy. Preliminary findings. \\ Arch Gen Psychiatry 1998 Sep;55:816-20.
}

\section{Question}

In patients who have recurrent depression after successful antidepressant treatment, is cognitive behavioural therapy (CBT) effective for reducing residual symptoms and relapse rates?

\section{Design}

Randomised controlled trial with 24 months of follow up.

\section{Setting}

An affective disorders programme in Bologna, Italy.

\section{Patients}

45 patients were enrolled, and $40(89 \%)$ were analysed (mean age 47 y, 60\% women). Patients had major depressive disorder according to the Research Diagnostic Criteria for a Selected Group of Functional Disorders with a global severity score of $\geqslant 7 ; \geqslant 3$ episodes of depression; $\leqslant 2.5$ years between the previous depressive episode and onset of the current episode; $\leqslant 10$ weeks of remission between the index episode and the previous depressive episode; and a successful response to antidepressants. Exclusion criteria were any medical illness; and history of manic, hypomanic, or cyclothymic features; antecedent dysthymia; substance abuse; or personality disorder.

\section{Intervention}

Patients were allocated to CBT $(n=20)$ or clinical management $(\mathrm{CM})(\mathrm{n}=20)$. Antidepressant drugs were tapered (at the rate of $25 \mathrm{mg}$ of amitriptyline hydrochloride [or its equivalent] every other week) and stopped. CBT and CM were given in 10 sessions of 30 minutes once every other week. CBT involved non-standard components of lifestyle modification and wellbeing therapy. CM consisted of monitoring medication tapering, reviewing the patient's clinical status, and providing support and advice, as necessary, without using exposure strategies, diary work, or cognitive restructuring. 2 independent assessors checked 8 randomly selected taped sessions and correctly identified all sessions.

\section{Main outcome measures}

Residual symptoms assessed by the Paykel Clinical Interview for Depression (CID), and rate of relapse.

\section{Main results}

Patients in the CBT group had fewer residual symptoms after treatment (mean CID score decrease 6.8, $\mathrm{p}<0.001$ ), and patients in the CM group had no changes in residual symptoms. Patients in the CBT group had fewer relapses than those in the CM group $(p<0.001)$ (table). Mean relapse free survival times were 92 weeks for the CBT group and 62 weeks for the CM group $(\mathrm{p}<0.001)$.

\section{Conclusion}

In patients with recurrent depression after successful drug treatment, cognitive behavioural therapy and tapered doses of antidepressants led to fewer residual symptoms and relapses than did clinical management and tapered doses of antidepressants.

CBT v CM with drug tapering for recurrent depression after successful drug treatment*

\begin{tabular}{lllll}
\hline Outcome at 2 years & CBT & CM & RRR (95\% CI) & NNT (CI) \\
\hline Relapse & $25 \%$ & $80 \%$ & $69 \%(37$ to 86$)$ & $2(2$ to 5$)$
\end{tabular}

*CBT $=$ cognitive behavioural therapy; $\mathrm{CM}=$ clinical management. Other abbreviations defined in glossary; RRR, NNT, and CI calculated from data in article.

Sources of funding: Istituto Superiore di Sanità and Ministero dell Università e della Ricerca Scientifica e Tecnologica.

For correspondence:Dr G A Fava, Department of Psychology, University of Bologna, Viale Berti Pichat 5, 40127 Bologna, Italy. Fax +39051243086 . (commentary continued from page 44) both apply. Few clinicians would expect such a brief intervention to retain effectiveness over 6 years when other psychosocial factors, such as subsequent life events, become more influential. Equally, the absolute numbers of patients relapsing in each arm of the trial on the 3 measurement occasions are so small $(3,7$, and $10 v 7,14$, and 15 patients for CBT and $\mathrm{CM}$, respectively) that before we can draw firm conclusions, these interesting findings should be replicated by a different group using a larger sample size.

The study published in the Arch Gen Psychiatry gives another indication that it may be worth considering a structured psychological therapy approach for those patients at highest risk of relapse: people with recurrent major depression. This study reports on a different cohort of patients from Bologna who received an idiosyncratic and rather eclectic form of CBT which included advice on lifestyle changes and a form of "well being therapy." All patients were treated by a single therapist. It is hard to say whether the good results are attributable to CBT as such, to this specific combination of therapeutic ingredients, or to the therapeutic talent of this particular psychiatrist. Disaggregating the effects of treatment, therapist, patient characteristics, and therapeutic alliance in psychotherapy research is notoriously difficult, and this preliminary study wisely leaves these questions alone. Readers also need to note that the results are applicable only to people with no history of person- ality disorder or dysthymia. The study is important, however, because it adds to gathering evidence that long term psychopharmacology, unacceptable to some patients, is not the only approach to prevent relapse in these common conditions.

Glenys Parry, DipClinPsych, PhD, CPsychol, FBPsS

Community Health Sheffield NHS Trust and University of Sheffield Sheffield, UK

1 Shea MT, Elkin I, Imber SD, et al. Arch Gen Psychiatry 1992;49:782-7.

2 Frank E, Kupfer DJ, Perel JM, et al. Arch Gen Psychiatry 1990;47:1093-9.

3 Blackburn IM, Moore RG. Br J Psychiatry 1997; 171:328-34.

4 Gloaguen V, Cottraux J, Cucherat M, et al. J Affect Disord 1998;49:59-72.

5 Gortner ET, Gollan JK, Dobson KS, et al.J Consult Clin Psychol 1998;66:377-84. 